\title{
Social disparities and gender gap in STEM learning: insights from a cultural landscape
}

\author{
Quan-Hoang Vuong \\ Centre for Interdisciplinary Social Research, Phenikaa University \\ Yen Nghia, Ha Dong district, Hanoi 100803, Vietnam \\ hoang.vuongquan@phenikaa-uni.edu.vn \\ Viet-Phuong La \\ Centre for Interdisciplinary Social Research, Phenikaa University \\ Yen Nghia, Ha Dong district, Hanoi 100803, Vietnam \\ phuong.laviet@phenikaa-uni.edu.vn \\ Manh-Toan Ho \\ Centre for Interdisciplinary Social Research, Phenikaa University \\ Yen Nghia, Ha Dong district, Hanoi 100803, Vietnam \\ toan.homanh@phenikaa-uni.edu.vn \\ Phuong-Hanh Hoang \\ Vietnam National Institute of Educational Sciences
}

101 Tran Hung Dao street, Hoan Kiem district, Hanoi 100000, Vietnam

hoangphuonghanh.hph@gmail.com

Working Paper No. PKU-1906

Hanoi, June 10, 2019

\begin{abstract}
:
This study reports the effects of socioeconomic status (SES) and gender on secondary students' academic results. Bayesian statistical analyses of 4967 observations show that gender, family size as well as parental education and occupation all contribute to students' performance in STEM subjects. Female students are reportedly less vulnerable to SES effects than males. The results provide cultural insights into the role of SES and policy implications to bridge the social gap in education.
\end{abstract}




\title{
Social disparities and gender gap in STEM learning: insights from a cultural landscape
}

\author{
Quan-Hoang Vuong, Viet-Phuong La, Manh-Toan Ho, Phuong-Hanh Hoang
}

Lifting labor productivity is the key solution for economic growth in the coming era with seamless technology advancement. One of the top educational foci towards a global economy is, therefore, STEM education, which aims to foster learners' skills concerning the areas of Science, Math, Engineering and Technology. Studies examining factors affecting students' cognitive development and academic aptitudes including STEM domains have identified the important role of socioeconomic background including birth order (1), family income (2), and parental educational level $(3,4)$ and occupation (5).

In general, researchers suggest that parenting styles and parental involvement, underpinned by the household socioeconomic status (SES), have impacts on children's cognitive development and STEM learning aptitude in particular. Social disparities in academic performance also include gender gap, which suggests that the effects of SES could vary depending on gender of students (6).

Vietnam with its high economic growth rates and top position in the PISA ranking of average scores in Math, Science and Reading provides an exotic case of the relationship between SES and STEM education. Despite the low national average income, Vietnam is among the countries with largest share of household contribution to total education spending and highest level of effectiveness of public expenditure on education (7). Considering the country's culture which values scholarship and growth mindset, examination of demographic factors and students' perfomance in STEM domains would enlighten the intertwinning between SES and academic achievement in similar transitioning sociocultural contexts.

This research utilized a dataset of 4967 observations of rural junior high school students from Northern Vietnam. A Bayesian network model (Fig. 1a) was simulated using R statistical software and the package bayesvl (8) to analyze different measures of SES and their effects on students' STEM results.

Figure 1. a) The designed model; Posterior coefficients of b) gender, and c) number of children and only child. 

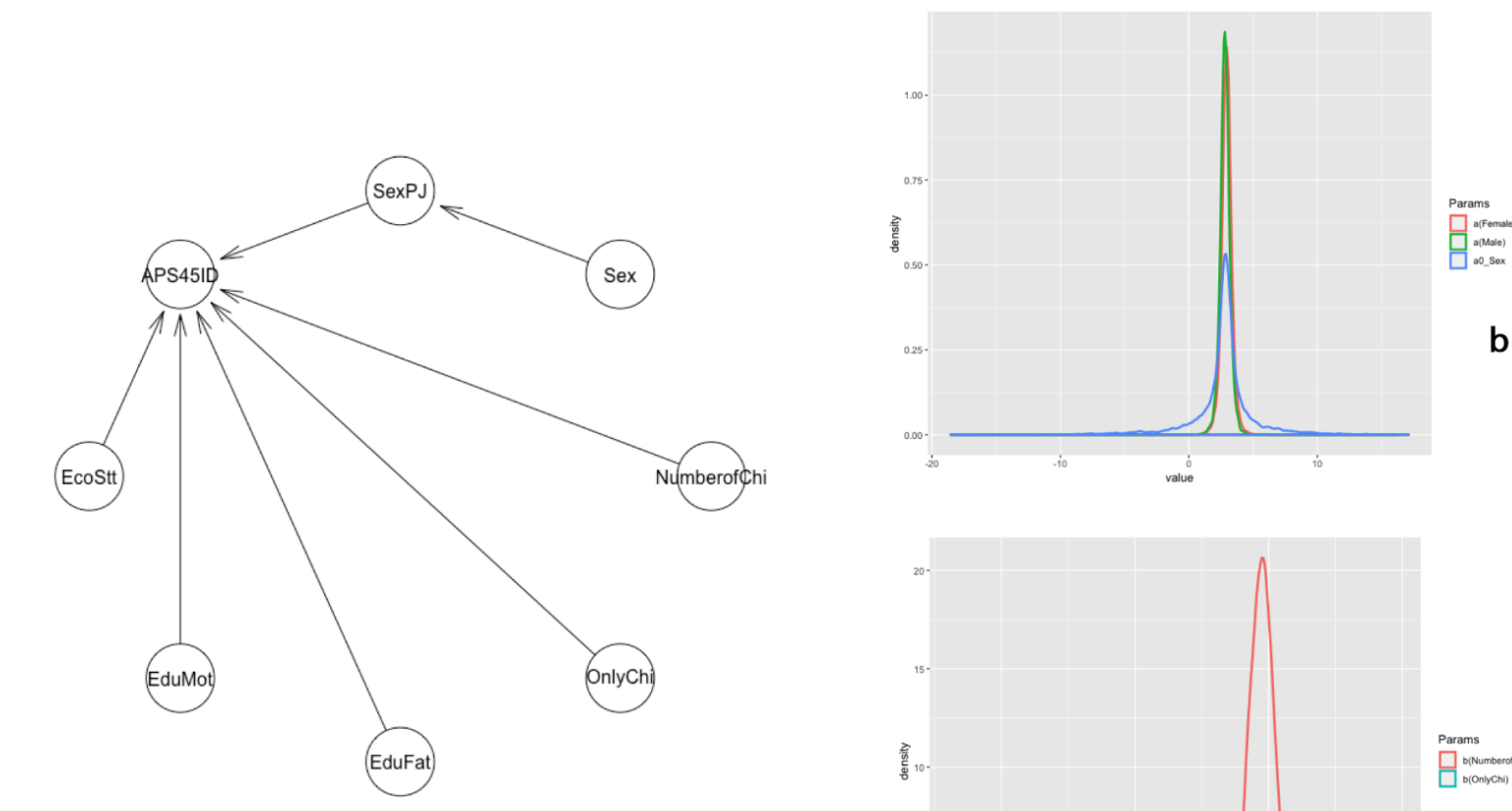

a

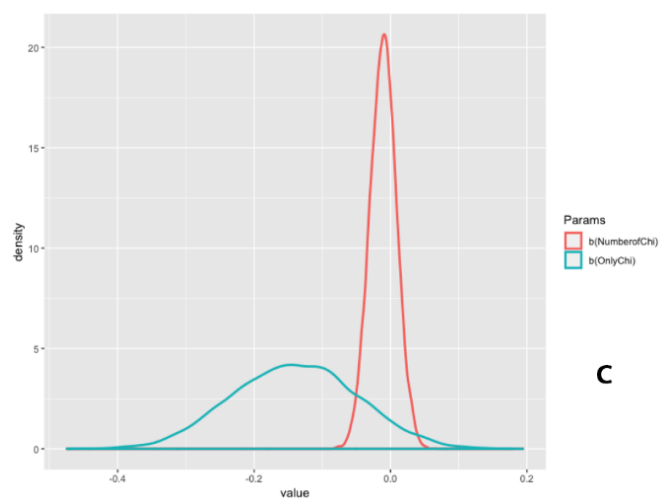

Firstly, analysis shows that gender has little association with the STEM results (Fig. 1b). Both genders yield positive values as illustrated, however, when examining the effect of gender in line with parental occupation, female students are likely to outperform their male peers in STEM subjects, even more so when their parents are labors (Fig. 2b). In a Confucian society, men would have more opportunities and privileges than women. Nevertheless, the findings reveal a "reverse gender gap" where girls are as or even more academically capable than boys in terms of test scores and transition to higher education $(9,10)$. It could be due to the fact that boys face higher pressure of joining the labor market during adolescence as they are expected to shoulder the responsibility of earning for the family. Gender norms also suggest that boys have the upper hand in the labor market to find better paid jobs than girls. This could explain the higher motivation for learning and pursuing higher levels of education of female students, reflected by their outscoring in test results reported by some nationwide education investigations (11). In other words, the reverse gender gap in Vietnam education might result from the reality that females are on the track to strive more for the same outcomes compared to males.

Secondly, the effect of family size was found discernible (Fig. 1c). Students having one sibling only seem to be of advantage compared to those with more than two siblings or without any. This is in line with previous literature that family size is detrimental to intellectual development of children since parental resources are finite and become diluted with the expansion of sibship size (12). However, inconsistent with existing studies on data in Western countries that the only child benefit from full access to parental financial and interpersonal resources, we found that being the only child in a rural family of a developing country is rather a disadvantage. First, the only child has neither an older brother/sister who serves as an intellectual source to stimulate intelligence development nor a younger sibling who gives the opportunities to take on the role of a "teacher" (13). As tutoring involves the reorganization and deep level processing of knowledge, having one (and not too many) younger sibling 
is considered favorable to a child's cognitive ability. Furthermore, from a cultural perspective, parents and caregivers in Vietnam, like other Confucian societies, tend to be more concerned about physical needs and less disciplinary to their only child $(14,15)$. To add on the social aspect is the fact that parents with only one child in the countryside of Vietnam are more likely to leave home for job opportunities in urban areas, having their child taken care by other relatives (16). Thus, lack of parental care and support due to domestic migration might negatively affect the only children's levels of academic achievement.

Figure 2. Posterior coefficients of a) parents' education and economic status, and b) students' sex and parents' jobs. Prediction of students' academic results when parent's jobs change: c1) both are intellectuals, c2) either father or mother is labor, c3) both are labors.

$\mathbf{S}$
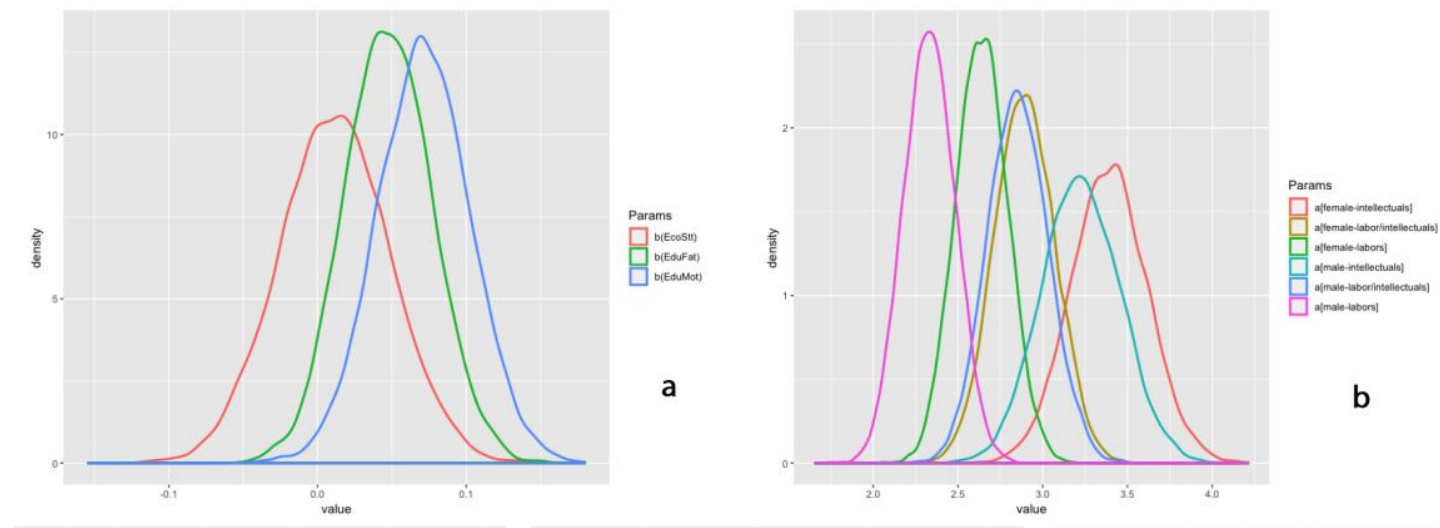

b
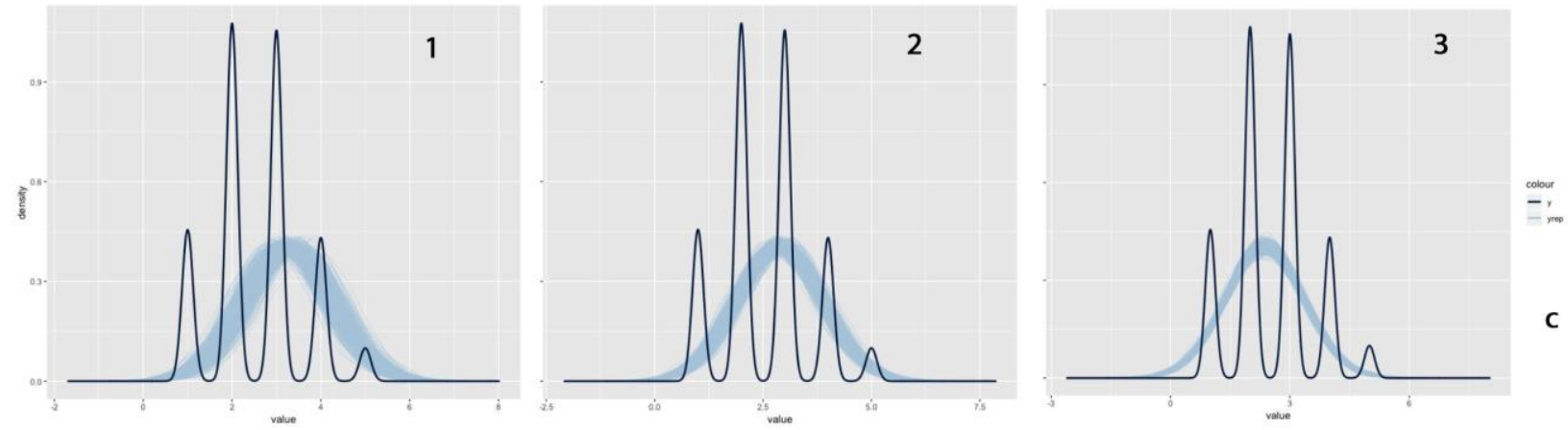

Thirdly, parents' education and occupational class could also account for students' academic achievement. Data analysis shows that parental educational level is positively associated with students' results (Fig. 2a). In terms of parents' occupation (Fig. 2b), students have the propensity to achieve better if their parents are intellectuals. In other words, students whose either or both parents are intellectuals tend to have better results than those born into labor families. The findings have hinted and predicted the effect that home environment and parenting styles might have on children's cognitive development in less developed economic regions (Fig. 2c). Intellectuals are generally seen as elite class in the Confucian culture who are better resourced to provide their children with a scholarly culture as well as impose strict disciplines, manners and etiquettes. All these would foster children's cognitive skills and cultural capital for academic success $(17,18)$.

Given the apparent effects of SES factors despite government efforts to ensure equitable education, deeper levels of intervention are critical such as enhancing school culture to narrow the SES gap in lower middle-income economies and promote education of scientific knowledge to the general 
public (19). In addition, given the low enrollment rates of female students in STEM majors at universities despite their equal capability demonstrated in secondary school, measures need to be taken to encourage female participation in tertiary level of STEM education to scale down waste of labor resources.

\section{Acknowledgements:}

This research was supported by Al for Social Data Lab, Vuong \& Associates, and SDAG, Phenikaa University (Hanoi, Vietnam).

\section{Author contributions:}

Q.H.V. designed research; Q.H.V. and V.P.L. performed research; Q.H.V. and V.P.L. analyzed data; and Q.H.V., M.T.H. and H.P.H. wrote the paper.

\section{Data depositions:}

The dataset is available from OSF (https://osf.io/cp35h/), and CRAN 'bayesvl' R package (https://cran.rproject.org/package=bayesvl). The code is provided on (https://github.com/sshpa/bayesvl/blob/master/LectureNotes/4.Model 4a\%20(stem).R) and graphs from https://github.com/sshpa/bayesvl/tree/master/LectureNotes/4.Graphs.

\section{References:}

1. V. J. Hotz, J. Pantano, Strategic parenting, birth order, and school performance. J. Popul. Econ. 28, 911-936 (2015).

2. Y. Xie, M. Fang, K. Shauman, STEM Education. Annu. Rev. Sociol. 41, 331-357 (2015).

3. K. G. Noble et al., Family income, parental education and brain structure in children and adolescents. Nat Neurosci. 18, 773 (2015).

4. K. S. Kendler, E. Turkheimer, H. Ohlsson, J. Sundquist, K. Sundquist, Family environment and the malleability of cognitive ability: A Swedish national home-reared and adopted-away cosibling control study. Proc. Natl. Acad. Sci. U.S.A. 112, 4612-4617 (2015).

5. M. W. Moakler Jr., M. M. Kim, College major choice in STEM: Revisiting confidence and demographic factors. Career Dev Q. 62, 128-142 (2014).

6. C. S. Rozek, R. C. Svoboda, J. M. Harackiewicz, C. S. Hulleman, J. S. Hyde, Utility-value intervention with parents increases students' STEM preparation and career pursuit. Proc. Natl. Acad. Sci. U.S.A. 114, 909-914 (2017).

7. S. Takaoka, Vietnam's human capital: Remarkable education success and future challenges. World Bank (2019).

8. V.-P. La, Q.-H. Vuong, bayesvl: Visually Learning the Graphical Structure of Bayesian Networks and Performing MCMC with 'Stan'. The Comprehensive R Archive Network (CRAN) (2019).

9. C. Rolleston, P. Iyer, Beyond the basics: Access and equity in the expansion of post-compulsory schooling in Vietnam. Int. J. Educ. Dev. 66, 223-233 (2019).

10. M. Thanos, P. Van, G. S. John, Puzzle me this? The Vietnamese reverse gender education gap. WIDER Working Paper 2018/166 (2018).

11. O. B. Azubuike, A. W. Little, Learning motivations, learning outcomes and gender in Vietnam. Young Lives (2019).

12. J. Blake, Family size and the quality of children. Demography 18, 421-442 (1981).

13. R. B. Zajonc, G. B. Markus, Birth order and intellectual development. Psychol. Rev. 82, 74-88 (1975). 
14. D. Davin, "The early childhood education of the only child generation in urban China" in Chinese Education Problems, Policies, and Prospects, I. Epstein, Ed. (Routledge, 2017), pp.42-65.

15. Q.-H. Vuong et al., Cultural additivity: behavioural insights from the interaction of Confucianism, Buddhism and Taoism in folktales. Palgrave Commun. 4, 143 (2018).

16. GSO, UNFPA, The 2015 national internal migration survey: Major findings. General Statistics Office \& United Nations Population Fund, Hanoi, Vietnam (2016).

17. J. Sikora, M. D. R. Evans, J. Kelley, Scholarly culture: How books in adolescence enhance adult literacy, numeracy and technology skills in 31 societies. Soc. Sci. Res. 77, 1-15 (2019).

18. M. D. R. Evans, J. Kelley, J. Sikora, Scholarly culture and academic performance in 42 nations. Soc. Forces 92, 1573-1605 (2014).

19. Q.-H. Vuong, The (ir)rational consideration of the cost of science in transition economies. Nat. Hum. Behav. 2, 5-5 (2018). 